\title{
A Brief Review of the FDA Dissolution Methods Database
}

\author{
I. E. Shohin1, D. Yu. Grebenkin 2,* , E. A. Malashenko³, Ya. M. Stanishevskii², \\ and G. V. Ramenskaya ${ }^{3}$ \\ ${ }^{1}$ Center of Pharmaceutical Analytics Ltd, 20, Nauchniy Proezd, Moscow 117246, Russia \\ 2 Institute of Biochemical Technology and Nanotechnology Peoples' Friendship University of Russia, 10-2, \\ Miklukho-Maklaya St., Moscow 117198, Russia \\ ${ }^{3}$ I.M. Sechenov First Moscow State Medical University, 8, Trubetskaya st., Moscow 119991, Russia
}

e-mail: igorshohin@yandex.ru

\section{ABSTRACT}

The FDA Dissolution Database was reviewed using the following criteria: dosage forms, apparatus, rotation/pulsation speed, dissolution media, sampling time points, and trends for special dosage forms. In July 2015, there were 1084 drug products in the database, more than $50 \%$ thereof in tablet form. The paddle (Apparatus 2 ) is the most common apparatus in the database and is recommended for 488 products (45\%). Rotation speeds listed in the database are 35-200 rpm for Apparatus 1 and 25-200 rpm for Apparatus 2. Deaerated or degassed water is recommended for 114 methods. The pH values for the most commonly cited dissolution media are in the range of 1-7.5; however, several dissolution methods have $\mathrm{pH}$ values that are out of physiological range $(\mathrm{pH} 12$ for celecoxib capsules, $\mathrm{pH} 9.5$ for glyburide tablets, $\mathrm{pH} 8.0$ for Rabeprazole Sodium Tablets, pH 7.8 for Glimepiride Tablets).

KEYWORDS: Dissolution; FDA; dissolution methods database; dissolution apparatus.

\section{INTRODUCTION}

I he dissolution profile test is one of the most useful methods used in different stages of the drug product lifecycle: pharmaceutical development, stability studies, interchangeability evaluation, routine quality control, and scale-up and post-approval changes. This test is suitable for a wide range of dosage forms such as tablets and capsules, transdermals, suspensions for internal use and for injection, suppositories, gums, chewing tablets, powders, vaginal inserts, implants, and others. The Food and Drug Administration Dissolution Database (1) is open access and is updated on a quarterly basis. There were 1084 drug products in the database at the time of search (July 2015). The database provides information on drug products in terms of International Nonproprietary Name (INN), dosage form, USP apparatus type, speed (rpm), dissolution medium, volume, sampling time points, and date of latest update. The structure of the FDA Dissolution Database reported was reviewed in more detail (2). The database is one of the most accessible, suitable, full, and informative resources on dissolution.

The aim of this paper was to make a brief review of the database in terms of dosage form, apparatus, rotation/ pulsation speed, and dissolution media.

\footnotetext{
*Corresponding author
}

\section{Dosage Forms}

The most common dosage forms in the FDA database are tablets and capsules (Figure 1). More than $65 \%$ of the products presented are in tablet dosage form. There are delayed-release tablets, coated tablets, enteric-coated tablets, orally disintegrating tablets (ODT), and chewing tablets. About $26 \%$ of the products are capsules, among which are the soft capsules, capsules with liquid contents, and capsules with delayed release.

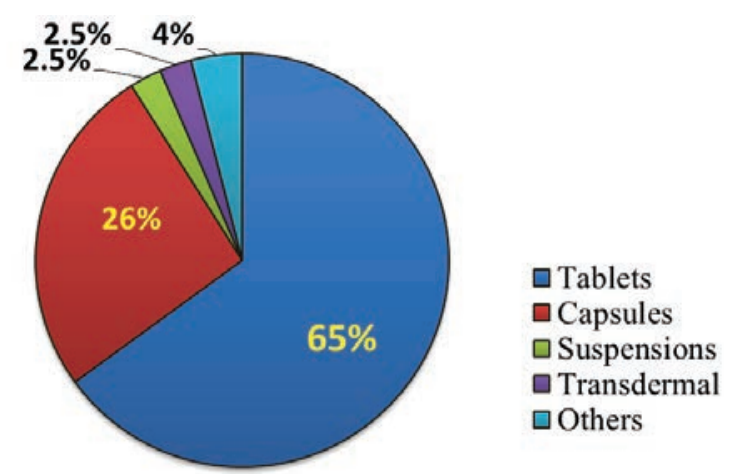

Figure 1. Dosage forms in the FDA database.

The FDA database contains dissolution methods for suspensions for internal and external use, injectable 
suspensions, and ophthalmic and otic suspensions. However, methods for such dosage forms are rather rare (about $2.5 \%$ of the database content). About $2.5 \%$ of the database describes the dissolution of transdermal dosage forms such as patches, films, and others. It also contains methods for suppositories, powders, pellets, vaginal rings, vaginal inserts, implants, gels, and chewing gum.

\section{Apparatus}

All USP dissolution apparatus (Apparatus 1-7) are listed in the dissolution methods database (3). The paddle (Apparatus 2) is the most common apparatus in the database. It is recommended for approximately $70 \%$ of the dissolution methods (Figure 2) and is considered the apparatus of choice for dissolution profile testing of immediate-release solid dosage forms. For capsule dosage forms, sinkers may be used to prevent floating. They include spiral, pronged, and basket sinkers. However, cone formation is an important disadvantage of the paddle. The disintegrated mass of a dosage form may settle at the bottom of the USP vessel where the agitation rate is minimal and form a cone of trapped drug particles, which leads to low dissolution rates.

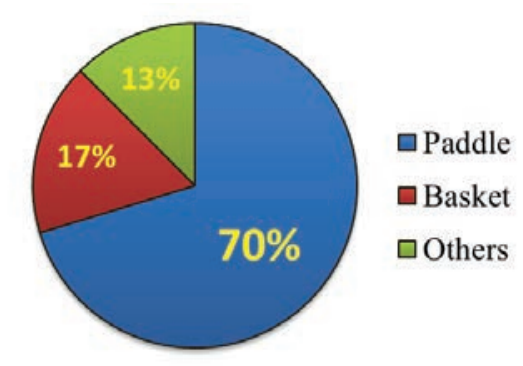

Figure 2. Frequency of apparatus cited in the FDA database.

Approximately $17 \%$ of dissolution methods (122 methods) recommend using the basket (Apparatus 1). This apparatus is commonly used for the dissolution of floating dosage forms. However, it has several disadvantages: disintegrated particles can clog the basket and small, disintegrated particles can fall out.

Apparatus 3 (reciprocating cylinder) is recommended for three extended-release tablets in the database: chlorpheniramine maleate, dexbrompheniramine maleate, pseudoephedrine sulfate. Apparatus 3 can be especially useful in cases where one or more $\mathrm{pH} /$ buffer changes are required in the dissolution testing procedure or when hydrodynamics can be directly influenced by varying the dip rate.

Apparatus 4 (flow-through cell) is recommended for only three methods: Betamethasone Acetate /
Betamethasone Sodium Phosphate Injectable Suspension, Medroxyprogesterone Acetate Injectable Suspension, and Methylprednisolone Acetate Injectable Suspension.

Apparatus 5 (paddle-over-disk) is used for dissolution of topical and transdermal dosage forms (sublingual film of buprenorphine $\mathrm{HCl} /$ naloxone $\mathrm{HCl}$, topical patch of diclofenac epolamine, transdermal film of estradiol, etc.).

Apparatus 6 (rotating cylinder) is recommended for seven methods that should be carried out to ensure the quality and performance of topical and transdermal drug products.

Apparatus 7 (reciprocating holder) is also used for transdermal delivery systems and a variety of dosage forms (e.g., intravitreal implant of dexamethasone).

The FDA database also refers to a number of noncompendial apparatus, such as an incubator shaker used to dissolve a vaginal ring that is not described in pharmacopoeias. There is also the chewing machine used for the analysis of nicotine chewing gum, which is described by the European Pharmacopoeia. For 254 products (about 23\%), the database does not state the test conditions but refers to relevant USP monographs. There are no methods mentioned for a number of drug products, and in those cases, the need of method development is noted. This is especially important for injectable dosage forms.

\section{Rotation Speed}

Rotation speed for Apparatus 2 (paddle) is $25-200 \mathrm{rpm}$ as listed in the database. The lowest value ( $25 \mathrm{rpm}$ ) is typical for suspensions (e.g., Ampicillin for Oral Suspension and Meloxicam for Suspension). The most rapid rotation speed for Apparatus 2 is listed in the dissolution method for Triptorelin Pamoate Injectable Suspension (200 rpm) and Fentanyl Citrate Lozenges (175 rpm). A typical paddle agitation rate is $50 \mathrm{rpm}$.

The rpm values of Apparatus 1 (basket) vary in the range of 35-200 rpm. The most common basket rotation speed is $100 \mathrm{rpm}$ (87 methods of 122). The basket is rotated at maximum speed $(200 \mathrm{rpm})$ in the dissolution method for Quetiapine Fumarate Extended-Release Tablets. The lowest speed for Apparatus 1 is $35 \mathrm{rpm}$, which is used for Pitavastatin Calcium Tablets, while $40 \mathrm{rpm}$ is recommended for Estradiol Vaginal Tablets.

The most common speed for Apparatus 5 and 6 (paddleover-disk and rotating cylinder, respectively) is 50 $\mathrm{rpm}$. The flow speed of the dissolution medium for the Apparatus 4 (flow-through cell) is in the range of 8-17 mL/ $\min$. 


\section{Dissolution Media}

The FDA Dissolution Database describes a large number of different media, from water or simple buffer solutions with different $\mathrm{pH}$ values to solutions with added surfactants, organic solvents, and enzymes.

The simplest choice for a dissolution medium is water. Deaerated or degassed water is recommended for 114 methods. The main disadvantage of water as the dissolution medium is its low buffering capacity. Use of water as a dissolution medium is also discouraged because test conditions such as $\mathrm{pH}$ and surface tension can vary depending on the water source and may change during the dissolution test itself due to the influence of the active and inactive ingredients (4). Therefore, it is important to monitor the $\mathrm{pH}$ during the test when water is used as the medium. However, water is still widely used for dissolution profile tests (about $10 \%$ of methods).

The most common dissolution media in the database have $\mathrm{pH}$ values in the range of 1-7.5 (i.e., 1.2, 2, 4, 4.5, 5, $6,6.8,7.2$, and 7.5). These $\mathrm{pH}$ values are found in the $\mathrm{Gl}$ tract and are considered physiologically relevant.

It is important to note that FDA database methods are primarily developed to determine in vitro dissolution profiles of drug products; therefore, these media may not reflect in vivo physiological conditions in some cases. Nowadays the agency is emphasizing the discriminatory power of the method. It does not have to mimic what happens in vivo, but it must be discriminatory for the critical quality attributes. For this reason, the FDA database describes eight media whose $\mathrm{pH}$ values are outside the physiological parameter range (e.g., pH 12 for Celecoxib Capsules, pH 9.5 for Glyburide Tablets, pH 8.0 for Rabeprazole Sodium Tablets and Proguanil Hydrochloride Tablets; pH 7.8 for Glimepiride Tablets, Azilsartan Kamedoxomil Tablets, and Tretinoin and Isotretinoin Capsules).

According to the database, several aqueous solutions of acids could be used as dissolution media. The most commonly used acidic media are based on hydrochloric acid: $0.1 \mathrm{M}, 0.01 \mathrm{M}$, and $0.001 \mathrm{M} \mathrm{HCl}$. Phosphoric acid $(0.01 \mathrm{M})$ and other acidic solutions may also be used.

Various buffer solutions (acetate, citrate, and phosphate) are recommended for dissolution testing in mildly low $\mathrm{pH}$. Dissolution media with high $\mathrm{pH}$ values are prepared by adding sodium hydroxide solution, borate buffers, and Tris buffers.
For water-insoluble or sparingly soluble drug products (BCS Class II or IV), the use of a surfactant (18.5\% of methods) such as sodium lauryl sulfate, Tweens (polysorbate 20 and 80), lauryl dimethylamine oxide (LDAO), Triton X, Brij 35 (polyoxyethylene lauryl ether), cetyl trimethyl ammonium bromide (CTAB), and others (Figure 3 ) is recommended. The most commonly used surfactant is sodium lauryl sulfate (SLS, SDS); its concentrations are reported in the range of $0.01-3 \%$ (Figure 4). Dissolution is the absorption-limiting factor for poorly soluble drug products; therefore, a dissolution test is particularly useful for such formulations (5).

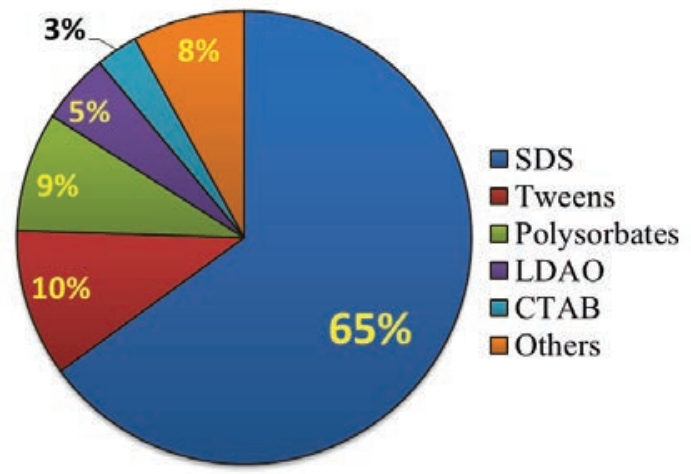

Figure 3. The most commonly used surfactants in the FDA database

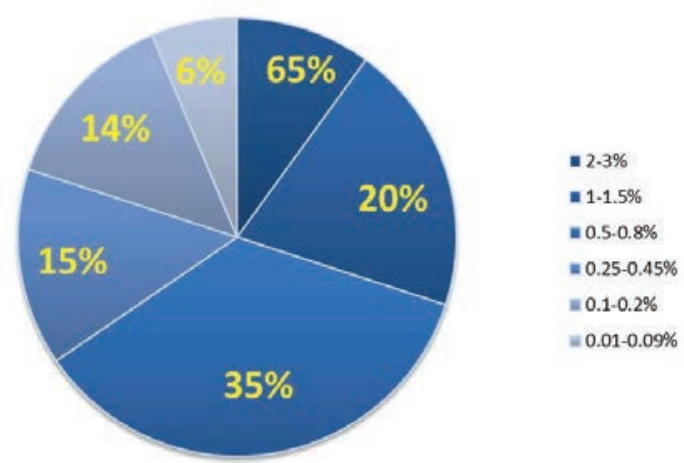

Figure 4. Media concentrations of sodium lauryl sulfate in the FDA database methods.

In some specific cases, the use of organic compounds is recommended. For instance, a medium containing $40 \%$ isopropanol is used in the method for Atovaquone Tablets. A medium containing $5 \%$ methanol is recommended for the injectable suspension of Triptorelin Pamoate. The necessity of an organic solvent for the dissolution test in these formulations is associated with the extremely low water solubility of atovaquone $(<0.2 \mu \mathrm{g} / \mathrm{mL})(6)$ and triptorelin pamoate. 
Dissolution media in the database may contain enzymes. The addition of enzymes may be considered, for example, for formulations containing gelatin in the outer layer (e.g., hard and soft gelatin capsules) (4). Pepsin solution in hydrochloric acid is used for the dissolution of dutasteride, loratadine, imipramine pamoate, enzalutamide, and emtricitabine capsules. Addition of pancreatin to the dissolution medium is recommended in the cases of dissolution of ibuprofen, bexarotene, and pseudoephedrine hydrochloride capsules.

There are 32 methods with simulated gastric fluid (SGF) or simulate intestinal fluid (SIF) as the dissolution medium. In several cases, these media may contain enzymes.

A change of dissolution medium $\mathrm{pH}$ values during the test occurs in 35 specifications of the FDA database. It is important for products and formulations for which dissolution testing has to simulate the passage of the dosage form between sections of $\mathrm{Gl}$ with different $\mathrm{pH}$ levels (e.g., enteric-coated tablets).

The volume of the dissolution medium is generally in the range of $500-1000 \mathrm{~mL}$ (Figure 5). The most commonly used volumes are $900 \mathrm{~mL}$ ( $56 \%$ of methods), $500 \mathrm{~mL}$ (12\% of methods), and $1000 \mathrm{~mL}$ (10\% of methods). Volumes of $750 \mathrm{~mL}$ and $600 \mathrm{~mL}$ often occur in methods that require a $\mathrm{pH}$ change of the dissolution medium.

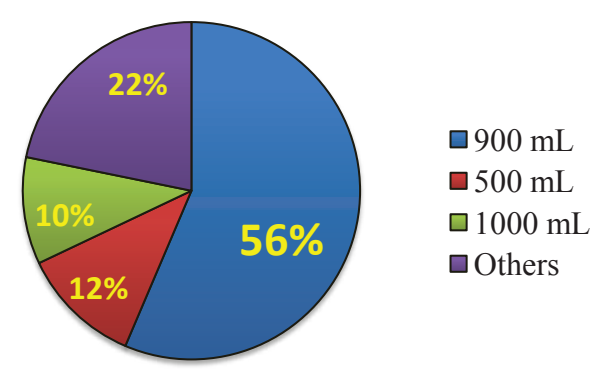

Figure 5. The most commonly used media volumes in the FDA database.

In specific cases, the FDA database recommends a volume less than $500 \mathrm{~mL}$ (e.g., $400 \mathrm{~mL}$ for Diclofenac Potassium Suspension). A decrease in dissolution volume is often dictated by the need to increase the sensitivity of quantitative determination methods and obtain dissolution profiles for low doses of drug products. For example, the database recommends Apparatus 1 and 2 that are specially adapted for small volumes of dissolution medium (60-200 mL) for buccal tablets and films of fentanyl at doses of 0.1-1.2 mg.

Most dissolution tests in the FDA database should be conducted at $37 \pm 0.5{ }^{\circ} \mathrm{C}$. The temperature of the dissolution medium is specified in cases where it differs from $37^{\circ} \mathrm{C}$. Generally, the temperature of the medium should be the same as the temperature of the application site of the product. The most commonly used temperature for topical dosage forms is $32{ }^{\circ} \mathrm{C}$. In cases of certain extended-release dosage forms where the product will remain at the site of application for a long time, generally for several months, it may be possible to reduce the duration of the dissolution test if the temperature of the test is greater than $37^{\circ} \mathrm{C}(7)$. The test temperature is 45 ${ }^{\circ} \mathrm{C}$ for Dexamethasone Implant (intravitreal), $40{ }^{\circ} \mathrm{C}$ for Mesalamine Suppository, and $38^{\circ} \mathrm{C}$ for Prochlorperazine Rectal Suppository. The temperature of the dissolution medium may also be less than $32{ }^{\circ} \mathrm{C}$. For instance, it is $25^{\circ} \mathrm{C}$ for oral suspensions and tablets of nitazoxanide and injectable suspensions of paliperidone palmitate.

\section{Sampling Time Points}

Sampling should be performed to obtain a full dissolution profile. For immediate-release products, the minimum number of sampling time points in the FDA database is three (e.g., 5, 10, $15 \mathrm{~min}$ for Risperidone Orally Disintegrating Tablet). However, typically five or more points are used (e.g., 10, 20, 30, 45, and 60 min in 59 methods; 5, 10, 15, 20, and 30 min in 43 methods; 10, 15, 20, 30, and 45 min in 25 methods; and 15, 30, 45, 60, and 90 min in 12 methods).

The most commonly used sampling time points for immediate-release dosage forms are $10 \mathrm{~min}(69 \%$ of methods), $20 \mathrm{~min}$ ( $60 \%$ of methods), $30 \mathrm{~min}$ (69\% of methods), and $45 \mathrm{~min}$ ( $53 \%$ of methods). Time points used less often are $15 \mathrm{~min}$ ( $37 \%$ of methods), $60 \mathrm{~min}$ ( $25 \%$ of methods), and others.

Most methods in the FDA database require sampling to begin within $10 \mathrm{~min}$ (319 methods), 5 min (210 methods), or 15 min (48 methods). An early start of sampling ( 2 or $3 \mathrm{~min}$ ) is typical for orally disintegrating tablets. The database requires sampling to start at 1 or $2 \mathrm{~min}$ for sublingual dosage forms.

The entire sampling process typically takes from 30 to 60 min, but sometimes it may take as little as 15 or as much as $180 \mathrm{~min}$ or more.

Sampling times for extended-release products should be considered separately and generally start with 0.5 or $1 \mathrm{~h}$ and take from 4 to $24 \mathrm{~h}$ including 5-12 time points. In the most difficult cases, dissolution may continue for 96 h (Naltrexone Hydrochloride Capsules, Granisetron Transdermal Film), 240 h (Dexamethasone Implant), 84 
days using an orbital shaker (Goserelin Acetate Implant, 10.8-mg strength), and 45 days using an incubator shaker (Estradiol Vaginal Ring).

\section{Special Dosage Forms}

Dissolution profile testing is suitable for a variety of novel or special dosage forms. Such dosage forms are represented in the FDA dissolution database by orally disintegrating tablets (ODT), suspensions, and topical and transdermal delivery systems. Some important characteristics of the dissolution profile test for special dosage forms may be noted.

The rotating paddle is the most commonly used method for ODTs. There are 19 methods for dissolution of ODTs that use a paddle and 3 methods that use a basket. A significant feature of these methods is an early first sampling point; it is often at less than $10 \mathrm{~min}$ (generally, 2-5 min).

The rotating paddle with a low stirring rate (25-30 rpm) is recommended in $25 \%$ of methods for the drug release testing of suspensions. Moreover, the use of deaerated water as dissolution medium is comparatively frequent (16\% of suspension methods).

The paddle-over-disk and the rotating cylinder are recommended for testing transdermal dosage forms (approximately 50/50). An important feature of these methods is that media $\mathrm{pH}$ values range from 4.5 to 6.8 , reflecting physiological skin conditions. For the same reason, the test temperature is typically set at $32^{\circ} \mathrm{C}(8)$.

\section{CONCLUSION}

Generally, the FDA dissolution method database can serve as a source of the most up-to-date information on various aspects of dissolution testing: apparatus, dissolution medium, rotation/pulsation speed, test conditions, and sampling time points. The database is especially useful for dissolution profile tests of generic drug products. Some of the most important advantages of the database are the quarterly update, open access, systematization of data, and ease of use. It should be emphasized that the database is a starting point for dissolution profile testing, and other conditions may be used with appropriate justification.

\section{CONFLICT OF INTEREST}

The authors declare that there is no conflict of interest.

\section{REFERENCES}

1. Dissolution Methods Database. U.S. Food and Drug Administration Web site. http://www.accessdata.fda.gov/ scripts/cder/dissolution/index.cfm (accessed June 17, 2016).

2. Shohin, I. E. FDA Dissolution Database is an indispensable tool for drug products developers ["Baza dannyh po rastvoreniju

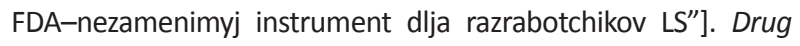
Dev. Reg. 2012, 1 (1), 3-13.

3. The United States Pharmacopeia and National Formulary USP 35-NF 31; The United States Pharmacopeial Convention, Inc.: Rockville, MD, 2013.

4. Dissolution Testing of Immediate Release Solid Oral Dosage Forms; Guidance for Industry; U.S. Department of Health and Human Services, Food and Drug Administration, Center for Drug Evaluation and Research (CDER), U.S. Government Printing Office: Washington, DC, 1997. http://www.fda.gov/downloads/ drugs/guidancecomplianceregulatoryinformation/guidances/ ucm070237.pdf (accessed June 17, 2016).

5. Hanson, R.; Gray, V. Handbook of Dissolution Testing, 3rd ed.; Dissolution Technologies, Inc.: Hockessin, DE, 2004; p 25.

6. Shaji, J.; Bhatia, V. Dissolution Enhancement of Atovaquone through Cyclodextrin Complexation and Phospholipid Solid Dispersion. Int. J. Pharm. Pharm. Sci. 2013, 5 (3), 642-650.

7. Brown, W.; Marques, M. Question and Answer Section. Dissolution Technol. 2014, 21 (2), 51. DOI: 10.14227/ DT210114P50.

8. Brown, C. K.; Friedel, H. D.; Barker, A. R.; Buhse, L. F.; Keitel, S.; Cecil, T. L.; Krämer, J.; Morris, J. M.; Reppas, C.; Stickelmeyer, M. P.; Yomota, C.; Shah, V. P. FIP/AAPS Joint Workshop Report: Dissolution/In Vitro Release Testing of Novel/Special Dosage Forms. AAPS PharmSciTech, 2011, 12 (2), 782-794. DOI: 10.1208/s12249-011-9634-x.

9. Waiver of In Vivo Bioavailability and Bioequivalence Studies for Immediate-Release Solid Oral Dosage Forms Based on a Biopharmaceutics Classification System; Guidance for Industry; U.S. Department of Health and Human Services, Food and Drug Administration, Center for Drug Evaluation and Research (CDER), U.S. Government Printing Office: Washington, DC, 2000. http://www.fda.gov/downloads/Drugs/ GuidanceComplianceRegulatoryInformation/Guidances/ UCM070246.pdf (accessed June 17, 2016). 